Reprod. Nutr. Dévelop., 1988, 28 Suppl. n¹, 167-168

\title{
Augmentation du pouvoir de rétention rénale de l'urée chez le mouton carencé en protéines : rôle du bassinet
}

\author{
A. CIRIO, R. BOIVIN
}

avec la collaboration technique de A. CHARLOT

Laboratoire de Physiologie.

Ecole Nationale Vétérinaire de Lyon.

B.P. 83, 69280 Marcy L'Etoile, France.

Summary. Sheep fed a low protein diet reduced renal urea excretion. An important fraction of urea perfused into the renal pelvis via the ureter, is reabsorbed through the pyelic epithelium.

Chez le mouton soumis à un régime carencé en protéines, l'élimination rénale d'urée est réduite (Schmidt-Nielsen et Osaki, 1958 ; Scott et Mason, 1970 ; Leng et al., 1985). Nous avons étudié l'intervention du pelvis rénal dans ce phénomène.

Matériel et méthodes. Nous avons utilisé deux lots de quatre brebis adultes (poids moyen $=40 \mathrm{~kg}$ ) qui avaient reçu, pendant un mois et pour $50 \mathrm{~kg}$ de poids vif, respectivement un régime standard $(P+)$ de $500 \mathrm{~g} / \mathrm{j}$ d'un mélange à $10,6 \%$ de matières azotées (200 g de luzerne déshydratée, $150 \mathrm{~g}$ de foin et $150 \mathrm{~g}$ d'amidon de maïs) et un régime hypoprotéique $(P-)$ de $500 \mathrm{~g} / \mathrm{j}$ d'un mélange à $8 \%$ de matières azotées (100 g de luzerne déshydratée, $200 \mathrm{~g}$ de foin et $200 \mathrm{~g}$ d'amidon de maïs). L'urémie, le débit urinaire, la quantité d'urée éliminée $\left(U_{u} V_{u}\right)$, le rapport urée urinaire/urée plasmatique $\left(U_{u} / U_{p}\right)$, le taux d'élimination de l'urée ( $T E_{\mathrm{u}}=$ proportion de l'urée filtrée retrouvée dans l'urine définitive), la clearance de l'urée $\left(C_{u}\right)$, la filtration glomérulaire $(F G)$ et le débit plasmatique rénal (DPR) ont été déterminés chez les deux lots d'animaux. Nous avons perfusé le bassinet par voie urétérale, avec des solutions aqueuses d'urée $1 \mathrm{M}$ et $3 \mathrm{M}$. Les perfusions ont été faites sur un rein en gardant l'autre comme témoin. Sur cinq périodes expérimentales de 30 min chacune ( $T$ = témoin, $1 \mathrm{M}=$ perfusion d'urée $1 \mathrm{M}, \mathrm{R}_{1}$ $=$ récupération $1,3 M=$ perfusion d'urée $3 M, R_{2}=$ récupération 2 ), nous avons déterminé la $C_{u}$ et le $T E_{u}$ de chaque rein.

Résultats et discussion. Les sujets $\mathrm{P}$ - ont eu une urémie inférieure à celle $\operatorname{des} P+(0,21 \pm 0,07$ contre $0,39 \pm 0,05 \mathrm{~g} / \mathrm{l}, \mathrm{p}<0,01)$, tout en renforçant leur pouvoir rénal de rétention de l'urée: réduction de $36 \%$ du $\operatorname{TE}_{\mathrm{u}}(0,38 \pm 0,19$ contre $0,59 \pm 0,28$ pour les $P+, p<0,05)$, de $56 \%$ de la $C_{u}(0,50 \pm 0,19$ contre $1,15 \pm 0,49 \mathrm{ml} / \mathrm{min} / \mathrm{kg}$ pour les $P+, p<0,01)$ et de $80 \%$ des quantités d'urée éliminées dans l'urine $(2,1 \pm 0,7$ contre $10,4 \pm 2,7 \mathrm{mg} / \mathrm{min}$ pour les $\mathrm{P}+$, $p<0,01)$. Les diminutions $d u$ débit urinaire $(7,6 \pm 0,5$ contre $14,5 \pm 7,9 \mu \mathrm{l} / \mathrm{min} / \mathrm{kg}$ pour les $P+)$ et du rapport $U_{u} / U_{p}(66 \pm 21$ contre $94 \pm 42$ pour les $P+$ ), ne sont pas significatives. Les valeurs de la $F G$ $(2,03 \pm 0,54(P+)$ contre $1,55 \pm 0,57(P-) \mathrm{ml} / \mathrm{min} / \mathrm{kg}$, N.S. $)$ et du DPR $(9,5 \pm 4,6(P+)$ contre $7,1 \pm 3,4(P-) \mathrm{ml} / \mathrm{min} / \mathrm{kg}, \mathrm{N} . \mathrm{S}$. $)$ indiquent que la 
diminution de ces paramètres chez les sujets carencés, est un phénomène mineur dans l'épargne de l'urée. Par contre, les perfusions du bassinet mettent en évidence un passage important de l'urée perfusée à travers l'épithélium pyélique. L'évolution du $T E_{u}$ pendant l'expérimentation est représentée sur la figure 1 (l'évolution de la $C_{u}$ est identique). Le $T E_{u}$ du rein perfusé en $3 \mathrm{M}$, chez les sujets $\mathrm{P}-$, devient négatif, ce qui traduit une rétention considérable d'urée. Le fait que ces modifications ne se produisent pas simultanément dans le rein non perfusé, indique que l'urée réabsorbée provient du perfusat. Cette propriété du bassinet a été développée par le régime hypoprotéique, puisque l'effet ne se retrouve pas dans les reins perfusés des $P+$. Le calcul montre que ce passage n'est pas dû à un accroissement artificiel du gradient urée urinaire-urée médullaire, lors de l'utilisation de l'urée en solution $3 \mathrm{M}$. L'élévation significative du $T E_{u}$ dans les périodes $R_{1}$ et surtout $R_{2}$, peut s'expliquer par la surcharge en urée de la médullaire rénale à la suite des perfusions, ce qui diminue la capacité de réabsorption tubulaire de l'urée (donc augmente le $T E_{u}$ ) pendant les périodes de récupération.

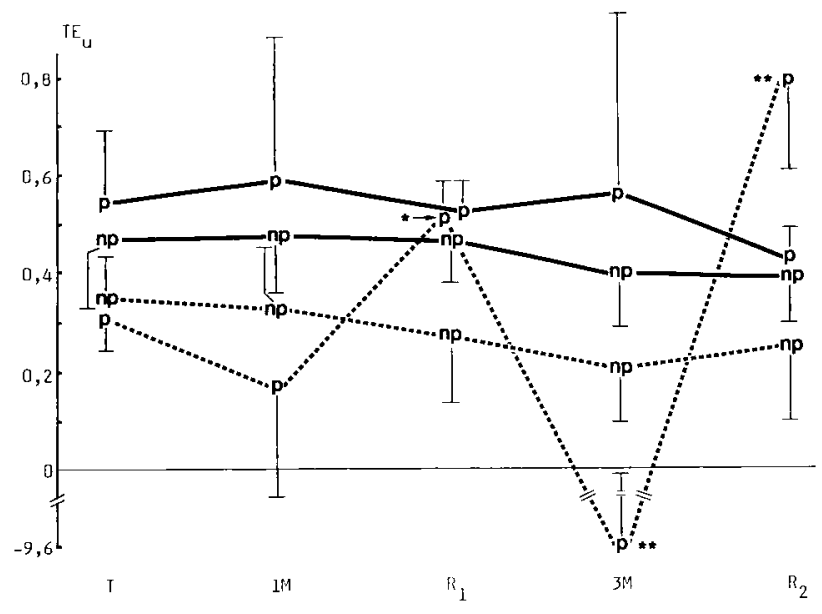

FIG. 1. - Evolution du taux d'élimination de l'urée $\left(\mathrm{TE}_{\mathrm{u}}\right)$ du rein perfusé $(\mathrm{p})$ et du rein non perfusé $(\mathrm{np})$, chez les moutons en régime standard (__ et en régime hypoprotéique (- . - - ) a a cours des cinq périodes expérimentales. Le test de signification est établi entre $T$ et chacune des quatre autres périodes $(n=4) .^{*}=p<0,05^{* *}=p<0,01$.

En conclusion, le bassinet joue un rôle non négligeable dans l'épargne d'urée chez les moutons carencés en protéines, en permettant une récupération accrue de l'urée à partir de l'urine pyélique.

Travail réalisé avec une subvention de I'I.N.R.A.

Leng L., Szanyiova M., Boda K., 1985. Physiol. bohemos/ov., 34, 147-154.

Schmidt-Nielsen B., Osaki H., 1958. Am. J. Physiol., 193, 657-661.

Scott D., Mason G. D., 1970 . Q.J. exp. Physiol., 55, 275-283. 